\title{
Evolution of a pre-exposure prophylaxis (PrEP) service in a community-located sexual health clinic: concise report of the PrEPxpress
}

\author{
Nicolo Girometti ${ }^{\mathrm{A}}$, Sheena McCormack ${ }^{\mathrm{A}, \mathrm{B},{ }^{*}, \text { Emma Devitt }}{ }^{\mathrm{A},{ }^{*}}$, Keerti Gedela ${ }^{\mathrm{A},{ }^{*} \text {, }}$ \\ Nneka Nwokolo ${ }^{\mathrm{A}, *}$, Sheel Patel ${ }^{\mathrm{A}, *}$, Tara Suchak ${ }^{\mathrm{A},{ }^{*}}$, Alan McOwan ${ }^{\mathrm{A}, *}$ \\ and Gary Whitlock ${ }^{\mathrm{A}, \mathrm{C}, *}$ \\ A56 Dean Street, Chelsea \& Westminster Hospital NHS Foundation Trust, W1D 6AQ, London, UK. \\ ${ }^{\mathrm{B}} \mathrm{MRC}$ Clinical Trials Unit at University College of London (UCL), Aviation House, 125 Kingsway, \\ WC2B 6NH, London, UK. \\ ${ }^{\mathrm{C} C o r r e s p o n d i n g ~ a u t h o r . ~ E m a i l: ~ g a r y . w h i t l o c k @ c h e l w e s t . n h s . u k ~}$
}

\begin{abstract}
Screening and treatment of sexually transmissible infections, including HIV, are free in the UK nations; preexposure prophylaxis (PrEP) became free in England in October 2017 through the PrEP Impact trial. Doctor-led PrEP clinics started at 56 Dean Street in September 2015, with the drug purchased privately at full price. The service was expanded to other staff to support initiation and monitoring of increasing numbers of attendees purchasing PrEP from online pharmacies. Nonetheless, when the clinic was given a target of 1700 for the PrEP Impact trial, it was clear this could not be achieved in a timely manner through 56 Dean Street alone. To prepare for the trial, all staff with HIV testing competencies were trained in good clinical practice and trial-specific procedures, and a patient group directive was approved to facilitate nurse prescribing and dispensing. Electronic pro formas to capture eligibility for starting or continuing PrEP were adapted for the Dean Street Express clinic, with some information collected directly from service users using touch screens. These interventions, together with an update to the 2016 information leaflet developed by the community, enabled enrolment and follow-up of 1700 participants in 4 months. PrEP advice and monitoring were easily accommodated in the 56 Dean Street sexual health service, but did require additional training and approval for nurse prescribing and dispensing drug in order to achieve the target, which still fell short of the demand.
\end{abstract}

Additional keywords: HIV, prevention, PrEP monitoring.

Received 27 March 2018, accepted 21 August 2018, published online 25 September 2018

\section{Introduction}

\section{Context for model of care}

Sexual health care in England is commissioned by local authorities and delivered through approximately 200 sexual health clinics that are affiliated with National Health Service (NHS) hospitals, community health services or commercial organisations. HIV care is commissioned by NHS England and delivered in most of these clinics.

The 56 Dean Street (56DS) clinic, a satellite of Chelsea and Westminster NHS Foundation Trust, is a combined sexual health and HIV service located in Soho, central London, UK. The clinic provides free, confidential and comprehensive diagnosis and management of sexually transmissible infections (STIs), contraception and out-patient care to those diagnosed with HIV infection as well as viral hepatitis, referring to Chelsea and Westminster hospital if admission is required.
The service moved to its present location in 2009. Since opening, attendances for asymptomatic STI screening have risen year on year and, in order to increase clinic capacity, 56DS opened a new service, Dean Street Express (DSE), in February 2014. DSE is located at 34-35 Dean Street and was designed as a nurse-led service providing STI screening for people without symptoms. By automating steps in the patient pathway, the time to result notification and the time to treatment were reduced from a median of 7 days to $6 \mathrm{~h}$, and 9 to 2 days respectively. A full description of the model of STI screening at DSE is provided in Whitlock et al. ${ }^{1}$ Briefly, users access the service through computer touch screens, registering with their mobile telephone number, which generates a confirmation SMS text to access the service further. Users input their sexual history directly into the electronic patient record through the touch screen, and this informs the algorithm for the generation of labels, which informs the healthcare assistant

*These authors are part of the Dean Street Collaborative Group. 
which specimens are required. Testing for STIs comprises chlamydia and gonorrhoea testing performed by NAAT (nucleic acid amplification testing) on self-taken swabs and urine processed on-site in a GeneXpert Infinity machine (Cepheid, Sunnyvale, CA, USA) with a 90-min turnaround time. Blood tests for HIV, syphilis and, if needed, hepatitis $\mathrm{B}$ and $\mathrm{C}$ are collected after a trained staff member (a nurse or healthcare assistant) has reviewed the sexual history and provided risk reduction advice and referral to other services if appropriate. Blood tests are analysed in an off-site laboratory with a 6-h turnaround. All STI results are texted directly to the patient's mobile telephone from the clinic computer system, although positive HIV and hepatitis $\mathrm{C}$ results are communicated by a health advisor (an individual with experience in sexual health and HIV counselling, including adherence support, and with responsibility for recall and partner notification). Since the implementation of the DSE model of care, there has been a steady increase in daily attendances, with over 100000 chlamydia or gonorrhoea test notifications sent to attendees in 12 consecutive months in 2014-15. The volume of testing attendances increased by $59 \%$ over the same observation period, reaching a 5-day moving average of 241.0 per day at DSE. ${ }^{1}$

\section{Description}

Pre-exposure prophylaxis introduction in England and the local problem

Oral pre-exposure prophylaxis (PrEP) as coformulated tenofovir/emtricitabine is freely available on the NHS to those at risk in Wales and Scotland, but not in England. Nonetheless, there are several thousand PrEP users in England who have purchased PrEP from online pharmacies or directly from clinics.

56DS started to offer a PrEP service in September 2015, initially through consultant physician-led clinics to individuals who were able to pay for their drug at cost price. The consultants developed a PrEP guideline and provided individuals with an information sheet and collected consent. In October 2015, two UK-based websites were launched providing information about PrEP: www.Prepster.info and www.iwantprepnow (accessed 4 July 2018). The latter facilitated individuals to buy generic oral PrEP online for personal use. Due to the increase in numbers of clinic attendees disclosing their use of generic tenofovir/ emtricitabine as oral PrEP, it was necessary to expand the staff disciplines able to advise and monitor those sourcing generic PrEP. To assist with this, and ensure that the key messages were consistent whether delivered in the clinic or community, the first UK PrEP guide was produced in June 2016 by a collaboration of HIV journalists, UK doctors, charities and PrEP advocates. ${ }^{2}$ In parallel, the professional associations responsible for developing clinical guidelines for HIV and sexual health released a practical guide and simple flow chart describing the procedures and investigations at initiation of PrEP and quarterly follow-up. ${ }^{3}$

\section{PrEP initiation}

PrEP monitoring at 56DS can be provided by any member of staff who sees service users, most frequently nurses, but also doctors and healthcare assistants. At PrEP initiation, a baseline
PrEP pro forma in the electronic patient record is completed face to face during the consultation, prompting staff to ask about a past history of renal and bone problems, hepatitis B status and dosing preferences. Baseline tests for HIV (fourth-generation ELISA), serum creatinine and urine dipstick for assessment of proteinuria, as well as tests to determine hepatitis B status, if required, and a full screen for STIs, including chlamydia, gonorrhoea, syphilis and, where there is risk, hepatitis $\mathrm{C}$ are performed. Urine dipstick results are immediately communicated (if protein $1+$ or higher is found, a urine specimen for protein: creatinine ratio is obtained for analysis). Blood tests results for HIV, syphilis, hepatitis $\mathrm{B}$, hepatitis $\mathrm{C}$ and renal function have a turnaround time of $24 \mathrm{~h}$ and are reviewed by a dedicated team of nurses and healthcare assistants. In case of abnormalities, results are submitted to the team of doctors for review. Chlamydia and gonorrhoea tests results are delivered via text message to the patients within $6 \mathrm{~h}$ from sampling and, in the case of any positive result, an appointment for treatment is offered at the patient's first availability.

PrEP start is actively recommended in individuals with a frequent risk of exposure to HIV, even when this is recent, but a fourth-generation ELISA HIV test is performed on the day of PrEP start and offered 4 weeks later.

\section{PrEP follow-up}

Those continuing on PrEP are advised to return every 3 months for PrEP monitoring; at this visit, a follow-up pro forma provides prompts to check for ongoing need of PrEP, adherence, dosing preferences and to perform urinalysis, triggering additional investigations if needed. Additional support, practical and/or psychological, can be offered by an experienced team of health advisors who support people living with HIV who struggle with adherence. Anyone with a complex medical history requiring further medical assessment can be booked into a consultant physician-led appointment.

\section{Interventions to scale up for PrEPxpress}

In October 2017, NHS England began a 3-year implementation trial (the PrEP Impact trial; www.prepimpact.org, accessed 4 July 2018) with a target enrolment of 10000 at-risk participants attending the sexual health clinics in England and providing PrEP free of charge. The aim of the trial was to assess how many sexual health clinic attendees require PrEP, how many take it up and the duration of use by the study participants. 56DS was given a target of 1700 participants and, to accommodate this number, it was necessary to incorporate enrolment and follow-up at DSE at no extra costs for the commissioner. The additional costs for the hospital trust consisted in the purchase of urine dipstick testing strips as required for the routine management of PrEP users, the annual creatinine tests and modifications to the touch screens to incorporate PrEP questions. The clinic began enrolling participants to the study on 13 October 2017, with 1700 consenting participant reached within 4 months. Such high volume was achieved through engagement of the entire multidisciplinary team, including doctors, nurses, health advisors, the recall team and administrative staff. Most of the 100-strong staff team at 56DS completed study-specific 
good clinical practice (GCP) training, which allowed them to enrol consenting participants to the study. Clinical staff members were already proficient in providing information about PrEP and monitoring clinic attendees, but it was necessary to obtain approval for nurse prescribing and to complete training for this, as well as to arrange secure storage for the trial drug at DSE. Following the example set by the Australian EPIC (Expanded PrEP Implementation in Communities) trial, ${ }^{4}$ group information sessions were performed at 56DS at hourly intervals led by a study-specific GCP-trained staff member and a copy of the updated UK PrEP guide $^{2}$ provided to each of approximately 15 attendees.

Following the group session, each participant was interviewed individually face to face at DSE and all 15 were seen within a dedicated hour time slot the same day. During this initial consultation, an online study pro forma for participants initiating PrEP was completed and the study consent form signed. The baseline PrEP assessment and investigations followed the standard of care previously described, which required the addition of a second urine bottle and serum creatinine to the asymptomatic screening panel already in place at DSE. Once completed, oral PrEP was prescribed and 90 tablets dispensed according to the patient group directive (PGD), a written protocol that enables nurse prescribing in a hospital setting. Although this reflects standard prescribing practice for STIs in sexual health clinics, it was necessary for the hospital trust to seek legal advice about provision of PrEP in a clinical trial. The 3-monthly follow-up also followed the standard of care with 30, 60 or 90 tablets of oral PrEP dispensed according to need under PGD and recorded in the study drug accountability log. Individuals with a complex medical history, potential drug interactions, proteinuria or abnormal creatinine, or those who reported adverse events, were referred to a consultant physician-led PrEP Review clinic at 56DS. Otherwise, renal function was assessed annually through serum creatinine.

\section{Lessons learned}

Early diagnosis, treatment and partner notification are the cornerstones of control for STIs, which is why a free, confidential and open access service has been in place in England for over 100 years. We have automated steps in the patient pathway at 56DS through DSE to reduce the time to treatment of STIs, including HIV, to 2 days. ${ }^{5}$ PrEP was introduced informally in England through self-purchase of drugs from online pharmacies, and the clinicians and community worked together to ensure that this was safe by agreeing the key messages, expanding the screening panel to include serum creatinine and training additional staff to advise on initiation and monitoring of PrEP as demand grew. When the PrEP Impact study launched, further interventions were needed to scale-up the PrEP service, and to implement a system for nurse prescribing and dispensing drug at DSE. This was particularly challenging because the drug was provided through a clinical trial. After training most clinic staff in study-specific procedures, PrEP advice and clinical monitoring, obtaining approval for nurse prescribing in the context of a trial and adapting the touch screens to include PrEP questions, it was possible to enrol 1700 individuals in 4 months.

\section{Conflicts of interest}

The authors declare no conflicts of interest.

\section{Acknowledgements}

Sheena McCormack was supported by the Medical Research Council (MRC_UU_12023/23).

\section{References}

1 Whitlock GG, Gibbons DC, Longford N, Harvey MJ, McOwan A, Adams EJ. Rapid testing and treatment for sexually transmitted infections improve patient care and yield public health benefits. Int J STD AIDS 2018; 29: 474-82. doi:10.1177/0956462417736431

2 iBase. Guide to PrEP. 2016. Available online at: http://i-base.info/ guides/wp-content/uploads/2016/06/PrEP-leaflet-FINAL1.pdf [verified March 2018].

3 British HIV Association. PrEP guidance. Available online at: https:// www.bhiva.org/file/5b729cd592060/2018-PrEP-Guidelines.pdf [verified August 2018]

4 Zablotska IB, Selvey C, Guy R, Price K, Holden J, Schmidt HM, McNulty A, Smith D, Jin F, Amin J, , Cooper DA, Grulich AE. Expanded HIV pre-exposure prophylaxis (PrEP) implementation in communities in New South Wales, Australia (EPIC-NSW): design of an open label, single arm implementation trial. BMC Public Health 2018; 18: 210. doi:10.1186/s12889-017-5018-9

5 Byrne R, Cooper F, Appleby T, Chislett L, Freeman L, Kershaw E, Nwokolo N, Whitlock G, McOwan A. Can express treatment reduce onward transmission? In: Richardson D, editor. Proceedings of the BASHH Spring Conference; 1-3 June 2015; Glasgow, UK. Macclesfield: British Association for Sexual Health and HIV; 2015. [Abstract O19] 